UDC 621.391

\title{
INTELLIGENT CONTROL SYSTEM FOR WSN AND MANET
}

\author{
${ }^{1}$ Olexandr I. Lysenko, ${ }^{2}$ Miroslav K. Sparavalo, ${ }^{3}$ Valery A. Romaniuk, ${ }^{1}$ Stanislav V. Valuiskyi \\ ${ }^{1}$ Institute of Telecommunication Systems \\ Igor Sikorsky Kyiv Polytechnic Institute, Kyiv, Ukraine \\ ${ }^{2}$ Institute of Telecommunication Systems \\ Igor Sikorsky Kyiv Polytechnic Institute, New York, USA \\ ${ }^{3}$ Military Institute of Telecommunications and Informatization \\ Igor Sikorsky Kyiv Polytechnic Institute, Kyiv, Ukraine
}

Background. New hierarchical model of intelligent control system (ICS) for wireless sensor (WSN) and mobile adhoc networks (MANET) is proposed. There was formulated a mathematical statement of the problem of finding a rational WSN topology using unmaned aerial vehicals (UAVs), which consists of a problem of sensor localization in monitoring zone and a problem of UAV relay localization in the space for the organization of connected network with desired characteristics.

Objective. The aim of the paper is to develop the hierarchical model for intelligent agents interaction for the MANET or sensor class radio network control system development.

Methods. We use mathematical methods and algorithms (graph theory, vector optimization methods, fuzzy algorithms, dynamic programming algorithm) and computer simulation to describe hierarchical model of intelligent control system for WSN and MANET.

Results. The novelty of the model lies in using the graph theory to make a formal description of the functional subsystems of the network ICS (vertices of the graph) and their interaction processes (edges of the graph). Using the proposed model can accelerate and systemize the network design process considering their functioning environment and hierarchical structure of their ICS.

Conclusions. Using the intellectual agents technology and multiagent systems allows minimizing the service traffic and use network and node resources more efficiently.

Keywords: WSN; MANET; UAV; control; agent.

\section{Introduction}

One of the main problems during development of self-organized networks such as wireless sensor (WSN) and mobile ad-hoc networks (MANET) is creation of effective control system, which is able to cover a lot of management tasks such as topology management, routing management, radio resource management, security management, quality of service management etc. For this purpose it proposed hierarchical model of intelligent control system for WSN and MANET described in Section 1. The novelty of the model lies in using the graph theory to make a formal description of the functional subsystems of the network (vertices of the graph) and their interaction processes (edges of the graph). Using of proposed model can accelerate and systemize the network design process considering their functioning environment and hierarchical structure of their control system. Using of intellectual agents technology and multiagent systems allows minimizing the service traffic and use network and node resources more efficiently. One particular management task intended to plan the optimal topology of network nodes is described in Section 2. There was formulated a mathematical statement of the problem of finding a rational WSN topology using UAVs, which consists of a problem of sensor localization in monitoring zone and a problem of UAV relays localization in the space for the organization of connected network with desired characteristics. Rational WSN topology, built according to these principles, will reduce traffic and reduce energy consumption at the nodes, which in turn will increase network lifetime and reduce the total cost of its maintenance.

\section{Section 1. The hierarchical model of intelligent control system for WSN and MANET}

Dynamic nature of MANET and WSN with ground based and air based (e.g. UAVs) mobile nodes leads to necessity of hierarchical architecture of their control system (CS) (master nodes and slave nodes) $[1,2]$. CS nodes make decisions based on gathering and processing the large volumes of service information about both node and entire network status; it is impossible to have full network status 
information in real time, therefore CS should make decisions in uncertain conditions.

Network control process main requirement is that all management decisions for node and network resources must be carried out automatically by independent mobile nodes. Furthermore, during the management process every node's CS must consider not only its own goal function, but the goal function of all neighboring nodes [3], whose information is stored on the master node. In this scenario, MANET class radio network CS management decisions must be based on the intelligent ability to recognize and analyze different situations (on either node or network level).

Modern approach for intelligent node control system (ICS) design in view of the MANET functioning and mentioned above requirements is the use of the intelligent agents (IA) technology and multi-agent systems (MAS) [4]. Main feature of this technology is that an agent is considered as a hardware and software system that can make decisions in uncertain conditions. That is, IA and MAS can adapt to the changes in surrounding environment they interact with, even in the case when said changes are not defined in their behavior schemes.

There are many examples of IA and MAS used for gathering and processing information, as well as automatic management of different complex systems and processes [5]. But existing models of IA and MAS are designed using the intelligent methods that do not account for the MANET class network control features, and the lack of a method for designing corresponding models for ICS nodes delays the process of network development.

Therefore, the purpose of this article is to develop the hierarchical model for intelligent agents interaction for the MANET or sensor class radio network control system development.

\section{Initial Data for the model}

According to the concept [2], network ICS is an aggregation of interacting node ICS that are deployed using the IA technology [6]. In this case, IA stands for a software product able to act to achieve a given goal and in addition to the main features (reactivity, proactivity and sociality) has [7]:

Mobility - IA can carry out its functions on another node on behalf of the initiator node;

Intelligence - the main feature of IA, that presume its ability to self-learn in the process of the mobile node operations so that it can find optimal behavior patterns for cases not foreseen at the design phase.

Every IA of a node ICS is designed for a specific type tasks (performs different functions, depending on OSI model levels) (Fig. 1), can interact with other
IA for information exchange and make coordinated decisions, forming the executive layer of network ICS.

The coordination of IA operations on executive layer is managed by metaagents of node ICS. Multiple metaagents form the node layer of network ICS. In turn, coordination of metaagents' decisions of node ICS is managed by a master node. Any node of the network can be a master node, depending on its hardware or geographic location. As seen on Fig. 1, main management agents of the node ICS of the network can be distinguished as follows: functional agent, system agent, monitoring agent, diagnostics agent, foresight agent. Though, the quantity and composition of IA can vary drastically depending on the network node (mobile node, base station or sensor device) [8].

Metaagent takes care of coordination of IA operations to achieve common management goals using the management decision made by local agents and metaagents of neighboring nodes. Metaagent analyzes network information by communicating with neighboring nodes so that it is able to make a decision to provide a certain level of QoS.

System agent. Its main functions are: maintaining a database of neighboring node and network status (available resources), mobile node locations (topographic information); forming a knowledge base with rules of "behavior" of the given node under different circumstances; self-learning of the mobile node.

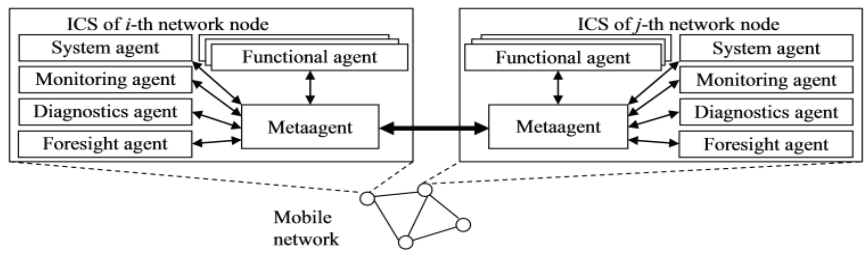

Fig. 1 Interaction of IA in the intelligent network control system

Monitoring agent - implements continuous monitoring of the network key performance indicators in real time; identifies different situation on the netowrk, determines current and potential problems; gathers and analyzes service information (statistics).

Diagnostics agent - determines, localizes and analyzes nod malfunctions; runs tests of main functions of all of the mobile node's modules.

Foresight agent - uses the rules and algorithms of network performance analysis on all its layers to make a forecast of node and network status in the near future.

Functional agents - implements control methods for every layer of OSI model: topology management, routing management, data streams management, 
queue management, message priority and security, spectrum allocation, power allocation etc.

Most of the aforementioned IA are stationary, they are located on the node permanently. But for some functions (network zone monitoring, information route planning, etc.) system agent can generate a mobile IA (MIA). MIA is relocated to another network node, collects (and processes if necessary) the information of the given node and returns to the source node with a report (or, if necessary, is relocated to a new node). MIA life cycle is illustrated on Fig. 2.

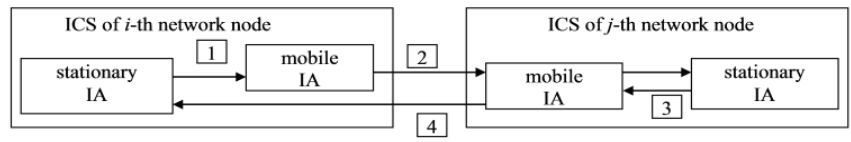

1 - node $i$ generates a MIA, 2-MIA relocation, 3 - data collection and processing, 4-return to the node $i$

Fig.2 MIA life cycle

Therefore, in view of the hierarchical concept of network ICS design [2] and aforementioned functional structure of node ICS with IA, formal description of MANET class radio network ICS can be presented as multiple IAs on different layers, that interact with each other by exchanging service information that is used to make management decisions. To achieve this we need to solve two problems: combine heterogeneous IA in the hierarchical network ICS and set up information exchange between IA in this structure. To solve these problems a hierarchical model of IA interaction is proposed, whose structure corresponds to the hierarchical network ICS design concept.

\section{Hierarchical model of IA interaction}

Formal description of the network ICS functional structure (with decentralized management) can be represented as a hierarchical IA structure with vertical relations between them. Given relations define the subordination of task that are resolved by IA at each layer [2]:

Zero (executive) layer - resolves management tasks according to the OSI model (routing, resource management, data streams management, security, etc.) by selecting the required values of node ICS subsystem parameters;

First (node) layer - consists of node ICS metaagents that coordinate the zero layer IA by selecting optimal set of management actions and their implementation sequence on all node ICS subsystems;

Second (network) layer - consists of the master node that corrects the goal functions of first layer meta-agents in view of network status, as w hole, or its part.
Using graph theory, we can picture the given functional structure as shown on Fig. 3. Located at the root of the tree is a master node subsystem, at the vertices that are one edge away from the root are subsystems $\left(I_{11}, U_{11}\right), \ldots,\left(I_{1 q}, U_{1 q}\right), \ldots,\left(I_{1 Q}, U_{1 Q}\right) \quad$ that represent Q meta-agents of node ICSs. Every mentioned subsystem of network ICS contains a control (identification) block I and management block U. In turn, every first layer subsystem $\left(I_{1 q}, U_{1 q}\right), q=\overline{1, Q}$ is connected to multiple functional subsystems of zero layer $P_{q r}, q=\overline{1, Q}, r=\overline{1, R}$, which are located on two edges distance from the root. These subsystems represent IA interaction processes of every functional subsystem of node ICS [9]. This interaction consists of service information exchange and management decisions of each IA.

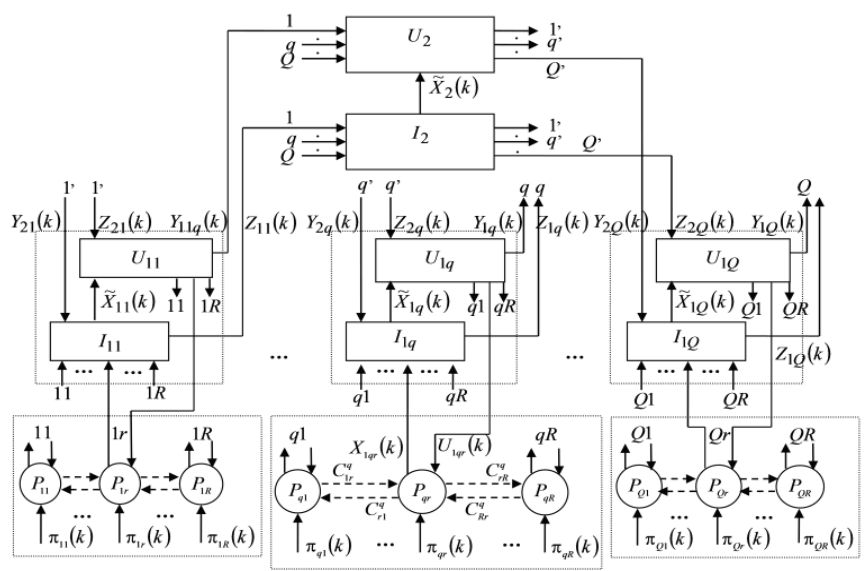

Fig.3 Hierarchical model of IA organization of network ICS

For q-th management subsystem of the first layer $\left(I_{1 q}, U_{1 q}\right), q=\overline{1, Q}$ let us denote the following:

$X_{1 q r}(k)$ - multiple state vectors of the qr-th IA, where the size of $X_{1 q r}(k)=\left\{x_{1 q r}^{a}(k)\right\}, a=\overline{1, a_{1 q r}}$ is $a_{1 q} \times 1$

$\tilde{X}_{1 q}(k)$ - multiple generalized estimated state vectors of q-th subsystem of the first layer (e.i. mobile node), where the size of $\widetilde{X}_{1 q}(k)=\left\{\tilde{x}_{1 q}^{a}(k)\right\}, a=\overline{1, a_{1 q}}$ is $a_{1 q} \times 1$;

$Y_{1 q}(k)$ - multiple management vectors of q-th subsystem of the first layer, which are directed to the upper layer management subsystem (master node), where the size of $Y_{1 q}(k)=\left\{y_{1 q}^{d}(k)\right\}, d=\overline{1, d_{1 q}}$ is $d_{1 q} \times 1$;

$Z_{1 q}(k)$ - multiple estimated state vectors of q-th subsystem of the first layer, that are directed to upper layer management subsystem (master node), where the size of $Z_{1 q}(k)=\left\{z_{1 q}^{d}(k)\right\}, d=\overline{1, d_{1 q}}$ is $d_{1 q} \times 1$; 
For the second layer management subsystem $\left(I_{2}, U_{2}\right)$ (master node), let us denote:

$\widetilde{X}_{2}(k)$ - multiple generalized estimated state vectors of the first layer subsystems (metaagents of the node ICS), where the size of $\tilde{X}_{2}(k)=\left\{\tilde{x}_{2}^{l}(k)\right\}, l=\overline{1, l_{r}}$ is $l_{r} \times 1=\left(\sum_{q=1}^{Q} a_{1 q}\right) \times 1$;

$Y_{2 q}(k)$ - multiple management vectors of control variables, that are sent to lower layer management subsystems (metaagents of the node ICS), where the size of $Y_{2 q}(k)=\left\{y_{2 q}^{d}(k)\right\}, d=\overline{1, d_{2 q}}$ is $d_{2 q} \times 1$;

$Z_{2 q}(k)$ - multiple management vectors of variable estimated states, that are sent to the lower layer management subsystem (metaagents of the node ICS), where the size of $Z_{2 q}(k)=\left\{z_{2 q}^{d}(k)\right\}, d=\overline{1, d_{2 q}}$ is $d_{2 q} \times 1$;

Finally, for qr-th subsystem of the zero layer $P_{q r}, q=\overline{1, Q}, r=\overline{1, R}$ let us denote:

$C_{r p}^{q}(k)$ - multiple connections vectors (service information exchange between IA and their management decisions), where $C_{r p}^{q}(k)=\left\{c_{r p}^{q^{m n}}(k)\right\}, m=\overline{1, m_{r}}, n=\overline{1, n_{r}}$ between r-th and p-th subsystems $(r, p=\overline{1, Q}, p \neq r)$;

$\Pi_{q r}(k)$ - multiple external effects vectors, that are been measured by r-th IA of the q-th mobile node, where the size of $\Pi_{q r}(k)=\left\{\pi_{q r}^{l}(k)\right\}, l=\overline{1, l_{q}}$ is $l_{q r} \times 1$.

Wherein, multiple vectors of q-th IA states $X_{q}(k)=\bigcup_{r=1}^{R} X_{1 r}(k)$ can be of different type depending on their state variables, which affect the channel quality and mobile node or network efficiency. Some of them are:

Network information load parameters vector

$$
\Lambda(k)=\left\|\Lambda_{1}(k), \ldots, \Lambda_{q}(k), \ldots, \Lambda_{Q}(k)\right\|^{T} ;
$$

Information messages delays vector

$$
\mathrm{H}(k)=\left\|\mathrm{H}_{1}(k), \ldots, \mathrm{H}_{q}(k), \ldots, \mathrm{H}_{Q}(k)\right\|^{T} ;
$$

Network radiofrequency environment parameters

$$
\aleph(k)=\left\|\aleph_{1}(k), \ldots, \aleph_{q}(k), \ldots, \aleph_{Q}(k)\right\|^{T} ;
$$

Network spectrum resources vector

$$
\mathfrak{I}(k)=\left\|\mathfrak{I}_{1}(k), \ldots, \mathfrak{I}_{q}(k), \ldots, \mathfrak{I}_{Q}(k)\right\|^{T} ;
$$

Network energy resources vector

$$
\mathfrak{R}(k)=\left\|\mathfrak{R}_{1}(k), \ldots, \mathfrak{R}_{q}(k), \ldots, \mathfrak{R}_{Q}(k)\right\|^{T} ;
$$

Hardware resources vector (processor, battery capacity, RAM, etc.)

$$
\mathrm{A}(k)=\left\|\mathrm{A}_{1}(k), \ldots, \mathrm{A}_{q}(k), \ldots, \mathrm{A}_{Q}(k)\right\|^{T} \text {, etc. }
$$

As shown in the model (Fig. 3), any q-th management subsystem of the first layer $\left(I_{1 q}, U_{1 q}\right), q=\overline{1, Q}$ can be characterized by:

Mapping that describes the object being managed (metaagent of q-th mobile node)

$$
\mathrm{O}_{1}^{(1)}: \widetilde{X}_{1 q} \times U_{1 q} \times C_{q p} \times \Pi_{q} \rightarrow Y_{1 q} \times Z_{1 q} .
$$

Mapping that describes the criteria used by q-th mobile node metaagent to determine the estimated state $V_{q}$ and control influence $W_{q}$

$$
\begin{aligned}
& \mathrm{O}_{2}^{(1)}: \widetilde{X}_{1 q} \times Z_{1 q} \rightarrow V_{q}, \\
& \mathrm{O}_{3}^{(1)}: U_{1 q} \times Y_{1 q} \rightarrow W_{q} .
\end{aligned}
$$

Mapping that describes the generalized information $\Phi_{q}$ that arrives to upper layer subsystem (master node)

$$
\mathrm{O}_{4}^{(1)}: Y_{1 q} \times Z_{1 q} \rightarrow \Phi_{q} .
$$

Mappings that determine the constraints of input variables vectors $\Theta_{q}$ and control influence vectors $\Psi_{q}$, respectively

$$
\begin{gathered}
\mathrm{O}_{5}^{(1)}: X_{1 q r} \rightarrow \Theta_{q}, \\
\mathrm{O}_{6}^{(1)}: U_{1 q} \rightarrow \Psi_{q} .
\end{gathered}
$$

Second layer subsystem $\left(I_{2}, U_{2}\right)$ can be characterized by:

Mapping that describes the formation of the generalized estimated states vector of the mobile network

$$
\mathrm{O}_{1}^{(2)}: \Phi \rightarrow \tilde{X}_{2},
$$

where $\Phi=\bigcup_{q=1}^{Q} \Phi_{q}$;

Mapping that describes the criteria used by the $\left(I_{2}, U_{2}\right)$ subsystem (master node) to determine the control influence destined for $\left(I_{1 q}, U_{1 q}\right), q=\overline{1, Q}$

$$
\mathrm{O}_{2}^{(2)}: U \times \tilde{X}_{2} \rightarrow W
$$

where $U=\bigcup_{q=1}^{Q} Y_{2 q}$;

Mappings that determine the constraints for generalized state and control vectors

$$
\begin{gathered}
\mathrm{O}_{3}^{(2)}: \widetilde{X}_{2} \rightarrow \Theta, \\
\mathrm{O}_{4}^{(2)}: U \rightarrow \Psi .
\end{gathered}
$$

The functioning of the ICS of all mobile network elements (mobile or sensor nodes, mobile base station or network control center) [9] can be described by time intervals, as follows:

$T_{1 q}$ - time interval for performing management and control tasks $(1-6)$ by metaagents of every node $\operatorname{ICS}\left(\left(I_{1 q}, U_{1 q}\right)\right.$ subsystems);

$T_{1 q}^{(2)}$ - time interval of generalized information transmission from metaagents $\left(I_{1 q}, U_{1 q}\right)$ to the master node $\left(\left(I_{2}, U_{2}\right)\right.$ subsystem $)$; 
$T_{2}$ - time interval for performing the control and management tasks $(7-10)$ by the master node.

The length of $T_{1 q}$ time interval is determined by the external influence vector $\Pi_{q r}(k)$ change rate, change of the control influences $Y_{2 q}(k)$ and $Z_{2 q}(k)$ from the master node $\left(I_{2}, U_{2}\right)$, and the change of interconnection matrix $C_{r p}^{q}(k)$ structure. The length of $T_{1 q}^{(2)}$ time interval is determined entirely by the methods and protocols of interaction between $\left(I_{1 q}, U_{1 q}\right)$ and $\left(I_{2}, U_{2}\right)$ subsystems, defined at appropriate levels of the OSI model.

Based on the information received by node ICS metaagents $\left(I_{1 q}, U_{1 q}\right), q=\overline{1, Q}$, the master node $\left(I_{2}, U_{2}\right)$ checks the restraints (9), (10) and calculates the values of the indicator in (8) with control influences $U(k)=\left\{U_{1 q r}(k)\right\}$, that are defined by subordinate node ICS on the previous time interval. If constraints are observed or a criterion has a deviation from the required value, a higher layer task is performed again, which defines the length of time interval $T_{2}$.

For a three layered network ICS (Fig. 3) the ratio between the aforementioned time intervals is as follows [9]:

$$
T_{2} \geq T_{1 q}^{(2)}, T_{2} \gg T_{1 q}, T_{1 q}^{(2)} \geq T_{1 q} \text {, for } \forall q=\overline{1, Q} .
$$

During those time intervals every element of the network ICS implements corresponding methods and algorithms of mobile network management, from mathematical methods and algorithms of link management (physical level of OSI model) to methods and algorithms of application level management (security management, power consumption management, QoS management, etc.).

\section{Decision making in the network intellectual control system}

In the general scenario, management decision making in the network ICS means providing a given quality of information exchange in MANET by determining the values of control variables of node ICS based on the analysis of current state of the radio network. But, as mentioned before, every node ICS is characterized by its own goal function, that is formed based on multiple factors:

Resources and hardware/software capabilities of the node, i.e. the totality of the devices for reaching the goal (RAM, processor performance, battery capacity, etc.);

Managed parameters: on physical level transmitter power, modulation, transmission rate, etc., on channel level - access protocol, on network level - routing method, on transport level - transfer method, etc.;
Uncontrollable parameters: set exchange protocols, topology dynamics, network size, interference level, etc.;

Requirements for information exchange quality for different types of traffic (data, voice, video and graphics).

It leads to the inability to achieve global optimization of the entire mobile network in the case of decentralized management environment and with presence of contradiction between the optimal node ICS awareness and the timeliness of control influences. Thereby, it was proposed in [10] to decompose the main goal of mobile network management to multiple simpler goals. To achieve this, in the design phase of node ICS a goal structure (GS) is formed as a graph, where the vertices are goals, and edges are the influences of achieving a goal in a subgoal (Fig. 4).

In the previous research it was shown, that in an uncertain environment, where a mobile network functions, to describe a situation and make a management decision by the subsystems of node ICS it is advisable to use the methods of fuzzy logic [11]. Therefore, the goal structure (Fig. 4) can be mathematically interpreted as a list of fuzzy management goals of different levels $L_{1}, \ldots, L_{k}$ are connected by $[10,12]$ :

$$
\begin{aligned}
& \mathrm{GS}=\left\{C_{1}, R_{2 m_{(1)}}\left\{C_{21}, C_{22}, \ldots, C_{2 m_{(2)}}\right\},\right. \\
& R_{3 m_{(2)}}\left\{C_{31}, C_{32}, \ldots, C_{3 m_{(3)}}\right\}, \ldots, \\
& \left.R_{k m_{(k)}}\left\{C_{k 1}, C_{k 2}, \ldots, C_{k m_{(k)}}\right\}\right\}
\end{aligned}
$$

where $C_{1}-$ global goal of the network ICS, that is determined by the master node; $C_{i l}, i=\overline{1, k}, l=\overline{1, m_{(i)}}$ - $l$-th subgoal of $i$-th level of the goal structure, that is determined by the metaagent of the corresponding node ICS; $\quad R_{i j}, \quad i=\overline{1, k}, \quad j=\overline{1, m_{(i-1)}}-$ fuzzy relationship between the lax advantage of the objects on the $i$-th level over every object at the upper $i$-1 level.

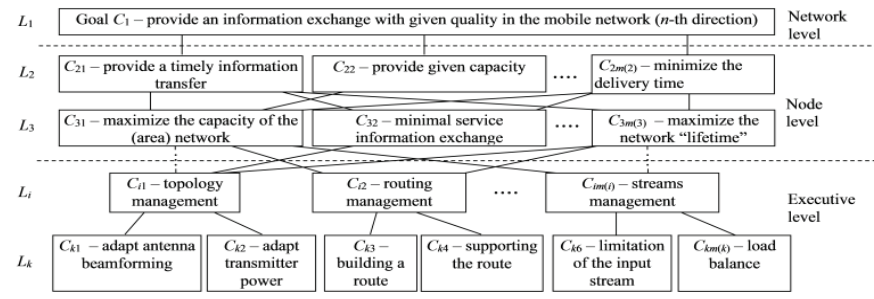

Fig.4. Fragment of the goal structure of the network ICS

If $R_{i j}$ describes the relationship only between the subgoals of neighboring levels, we should talk about a goal tree, otherwise the goal structure degenerates to a network. 
Let the goal system consist of $k$ levels and every $L_{i}$ level $i=\overline{1, k}$ consists of $m_{i}$ objects (for first level $\left.m_{1}=1\right)$ :

$$
L_{i}=\left\{C_{i 1}, C_{i 2}, \ldots, C_{i m(i)}\right\} \text {. }
$$

Goal structure (Fig. 4) can be described as a multiple of levels $L_{i}$ :

$$
\mathrm{GS}=\bigcup_{i=1}^{k} L_{i}=\bigcup_{i=1}^{k} \bigcup_{l=1}^{m_{i}} C_{i l}
$$

As seen on Fig. 4, different elements of the goal structure are united under a global goal $C_{1}$, that can be described as providing the information exchange with given quality in the network. As mentioned before, a binary fuzzy relationship of a lax advantage $R_{i j}$ is used to describe the relationship between global goal and lower level goals, that is given by a membership function $\mu_{R_{i j}}\left(C_{i l}, C_{i r}\right), \quad i=\overline{2, k}$, $j=\overline{1, m_{(i-1)}}, \quad l, r=\overline{1, m_{i}}$.

It should be noted, that depending on the hierarchy layer (Fig. 3) there can be two types of relationship:

"goal - subgoal" relationship - appear between the elements of the network and node layers (between master node and subordinate nodes of a mobile network or its area) and create a goalforming part of the GS;

"subgoal - means to reach the goal" relationship appear between elements of the node layer (metaagents of node ICS) and the elements of the executive layer (IA of corresponding functional subsystems) and create an implementing part of the GS.

And so, beginning with the second hierarchy layer (11), at every $i$-th layer there are as many fuzzy relationship of advantage $R_{i j}$ as there are objects at $i$ 1 level of GS. In the general case, these relationships can be described as a matrix:

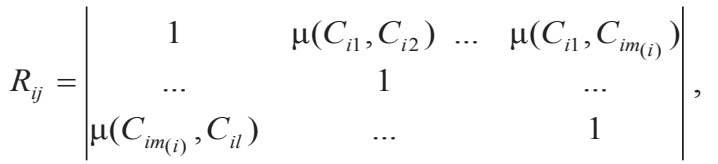

where $\quad \mu_{R_{i j}}\left(C_{i l}, C_{i r}\right) \in[0 ; 1], \quad i=\overline{2, k}, \quad j=\overline{1, m_{(i-1)}}$, $l, r=\overline{1, m_{i}}$.

As a result, tasks of decision making of the network ICS are reduced to receiving of the priority vector of the lower layer elements in relationship to the global goal - the element of the first layer. To cope with this task in [12] it is proposed to use a weighting procedure of the hierarchy analysis method or fuzzy relationship convolution algorithm.

\section{Section 1 conclusion}

Thereby, to respond to the features of the management in the MANET class mobile networks, the management system must have intellectual capabilities to recognize and analyze the situations in the radio network, and based on this, make management decisions to control the node and network resources. To design such management system, it is proposed to use the technology of intellectual agents and multiagent systems, that suggests that all subsystems of node ICS are implemented using multiple IA, that are defined by management functions depending on the level of the OSI network model.

To combine different IA in an intellectual network control system a hierarchical model of IA interaction was proposed in this article, whose essence lies in describing the network ICS as a hierarchical structure with vertical links, that indicate the subordination of management tasks.

The novelty of the model lies in using the graph theory to make a formal description of the functional subsystems of the network ICS (vertices of the graph) and their interaction processes (edges of the graph). Using the proposed model can accelerate and systemize the network design process considering their functioning environment and hierarchical structure of their ICS. Using the intellectual agents technology and multiagent systems allows minimizing the service traffic and use network and node resources more efficiently.

During future research a model for information resources organization of network ICS will be developed, to describe the circulation, processing and storage of the service information, which is used by the methods and protocols of corresponding subsystems for making management decisions in the mobile network.

\section{Section 2. The problem of finding a rational topology of wireless sensor networks using UAVs}

In recent years WSN are widely used in various fields of human life, ranging from automation processes of data collection on various parameters of the observed objects (buildings, industrial equipment and processes) to the health sector, security and defense [13-15]. However, the use of WSN for monitoring environmental parameters over large geographical areas requires specific network architecture, consisting of the following levels. The first level is a network of low-power wireless sensors, deterministic or randomly located in the large geographical area. The second level is a network of gateways, which represent more powerful sensor units (by CPU performance and battery 
capacity of nodes) and are intended to gather monitoring information from sensor nodes and transfer it to the processing centers and ensure the connectivity of WSN topology. In this regard, depending on the application (difficult terrains or infected areas, fighting field, etc.), gateways can be executed in the form of mobile robots or UAVs, which can form the third level of WSN.

One of the main problems in the organization of such complex hierarchical WSN for remote collection of information is the task of finding the optimal network topology, which consists of two partial problems. The first is the search of optimal placement of sensors for complete coverage of monitoring area. Second is the search of the optimal placement of UAV repeaters to organize a connected network with specified data transmission characteristics (throughput, delay, etc.). This article will consider the mathematical statements of these problems and possible methods for their solution respectively in the first and second section of the article.

\section{The problem of sensor localization in the monitoring zone}

Monitoring area of sensor (detection range) is usually represented as a circle of radius $r$ centered at the location of the sensor. Then the problem can be formulated as follows: it is necessary to place a minimum number of circles that provide coverage of whole monitoring area of potentially dangerous object. Usually monitoring area has arbitrary form that can be easily approximated by multiply orthogonal polygons.

The problem of covering a polygon is easily reduced to a simpler problem of covering a rectangular area with obstacles (RO) (Fig. 5). The output polygon is denoted by $P$, a limited rectangular region $-A$. Addition $A \backslash P$ will be interpreted as a set of fictitious obstacles. Next, we will work with RO. Specified and fictitious obstacles are shaded in the figure.

On the plane, we introduce a coordinate system $(O X, O Y)$ such a way that the coordinate axes match with the bottom and left sides of rectangular area $A$. Output information of the problem can be represented by the following set of data: $\{W, L, Z, r\}$, where $W$ and $L$ - width and length of a rectangle that will covered; $Z$ - obstacles that are given by set of rectangles $Z=\left\{Z_{1}, Z_{2}, \ldots, Z_{\mathrm{m}}\right\}$, where $m$ - number of rectangle obstacles. The sides of rectangle from $Z$ are parallel to the coordinate axes. $Z_{\mathrm{i}}=\left\{z_{\mathrm{xi}}, z_{\mathrm{yi}}, z_{\mathrm{li}}, z_{\mathrm{wi}}\right\}-$ rectangle, which simulates the obstacle, where $i=$ $1, \ldots, m .\left(z_{\mathrm{xi}}, z_{\mathrm{yi}}\right)-$ coordinates of the lower left corner of the rectangle $Z_{\mathrm{i}} ;\left(z_{\mathrm{li}}, z_{\mathrm{wi}}\right)$ - width and length of rectangle $Z_{\mathrm{i}}$. Thus polygon $A \backslash Z$ is needed to be covered by minimum number of equal circles $N$ of radius $r$.

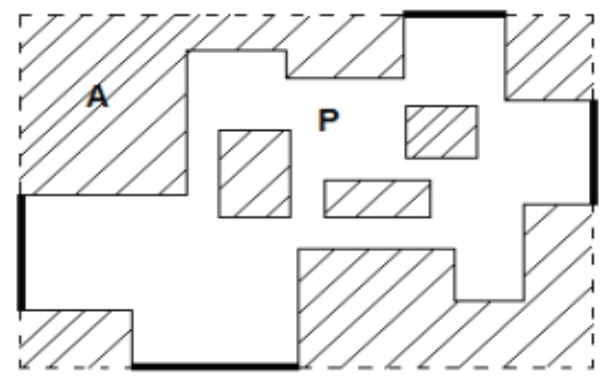

Fig.5 Monitoring area as polygon

Solution of the problem can be represented by a set of data: $C=\{N, X, Y\}$, where $N$ - number of covering circles in solution; $X=\left\{x_{1}, x_{2}, \ldots, x_{\mathrm{N}}\right\}, Y=$ $\left\{y_{1}, y_{2}, \ldots, y_{\mathrm{N}}\right\}-$ vectors of circle centers coordinates. Solution $C$ is acceptable covering if the following conditions $\Omega$ are performed:

1) Circles are inside of rectangle $A$ :

$x_{\mathrm{j}} \geq 0 ; y_{\mathrm{j}} \geq 0 ; x_{\mathrm{j}} \leq L ; y_{\mathrm{j}} \leq W \forall j=1, \ldots, N$.

2) Circle centers lie inside obstacles: at least one of the inequalities is performed:

$\left(x_{\mathrm{j}}-z_{\mathrm{xi}}\right)\left(x_{\mathrm{j}}-z_{\mathrm{xi}}-z_{\mathrm{li}}\right) \geq 0$ or $\left(y_{\mathrm{j}}-z_{\mathrm{xi}}\right)\left(y_{\mathrm{j}}-z_{\mathrm{xi}}-z_{\mathrm{li}}\right) \geq$ $0 \forall i=1, \ldots, m, j=1, \ldots, N$.

3) Area $A \mid Z$ is covered: if $\left(p_{\mathrm{x}}, p_{\mathrm{y}}\right)$ - an arbitrary point from $A \mid Z$, then $\exists j:\left(p_{\mathrm{x}}-x_{\mathrm{j}}\right)^{2}+\left(p_{\mathrm{y}}-y_{\mathrm{j}}\right)^{2} \leq r^{2}$.

Candidate solution $\mathrm{C}$ is optimal if the number of $N$ circles is minimum, i.e. $C_{\mathrm{opt}}=\arg \min _{X, Y \in \Omega} \mathrm{N}$.

Example of coverage is shown in Fig. 6. Obstacles in the figure are shown by dark color.

The solution of such problems is engaged by such scholars as L.F. Toth, S.N. Astrakov, Ye.A. Muhacheva, V.Y. Kuznetsov et al. Summarizing their experience it is possible to allocate the following heuristic-based approaches: heuristic block coverage, heuristic hexagonal coverage, pseudo hexagonal heuristics coverage, evolutionary heuristics and others. The experimental results showed that all the proposed algorithms can be used in solving the problem of optimal placement of sensors in monitoring zone, the choice of algorithm is dictated by the specific requirements of the task. The resulting solution $C_{\mathrm{opt}}$ is initial data for UAV repeaters placement algorithms described in the next section.

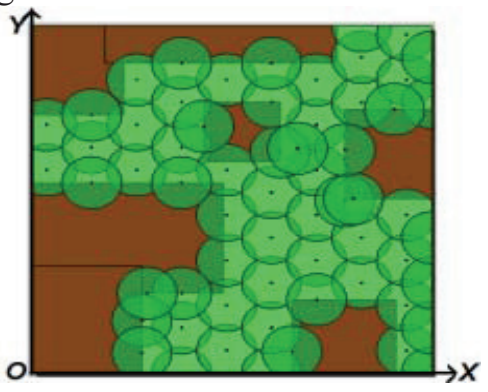

Fig.6 Coverage of the polygon by circles 


\section{The problem of UAV relays localization in the space}

Let's consider an example of WSN architecture using UAV (Fig. 7). WSN consists of a set of $N$ sensors, end devices (ED), located with the coordinates $\left(x_{i}, y_{i}\right), i=\overline{1, N}$, in some limited area with size $r$, which is often called by sensor field. In order to collect data from sensors and transmit them to the gateway it is used a set of $K$ relays (routers) based on miniature UAVs located at a height $h$ with the coordinates of the projection $\left(x_{0 k}, y_{0 k}\right), k=\overline{1, K}$ and radio coverage radius $R$. If sensor is located in the UAV radio coverage, data via repeaters network get to gateway and then via global networks to the data processing center (DPC). For data it can be used one of existing network protocols such as IEEE 802.15.4/ZigBee.

Under the network topology we mean a set of geometric location of nodes and the probability of use the communications between them to deliver messages: $\left(\left\|R_{i}\right\|,\left\|p_{i j}\right\|\right), i, j=\overline{1, N}$, where $\left\|R_{i}\right\|$ is a set of WSN nodes; $\left\|p_{i j}\right\|$ is a set of probabilities of using the communications between nodes. Thus, the WSN can be represented as a weighted directed graph consisting of a set of vertices (nodes) and edges (channels) (Fig. 8).

Construction the routes from ED to the gateway is realized by using a dynamic programming algorithm - the method of Bellman-Ford. Criterion function of this algorithm (Bellman function) defines the conditional cost of data flow transporting between adjacent nodes of route:

$$
\begin{gathered}
W=C_{m}+C_{r} \underset{\left\|R_{i}\right\|}{\min }, \text { where } C_{m}=\left\{\begin{array}{l}
C_{m}^{n o m}, \\
0
\end{array},\right. \\
C_{r}=\left\{\begin{array}{l}
\frac{U_{R X}^{*}+U_{T X}^{*}}{U_{\max }-U_{w}^{*}} C_{m}^{n o m},\left(U_{R X}^{*}+U_{T X}^{*}\right) \leq\left(U_{\max }-U_{w}^{*}\right), \\
\infty,\left(U_{R X}^{*}+U_{T X}^{*}\right)>\left(U_{\max }-U_{w}^{*}\right)
\end{array}\right.
\end{gathered}
$$

where $C_{m}$ is conditional cost of WSN node, which receives zero value with the repeated use of node in the topology (it allows minimizing the number of repeaters), $C_{m}^{\text {nom }}$ is conditional nominal cost of node, $C_{r}$ is conditional cost of relaying, $U_{R X}^{*}, U_{T X}^{*}$ is actual traffic through the node taking into account repeated relaying, $U_{\max }$ is maximum traffic through the node, $U_{w}^{*}$ is equivalent density of data flow emitted by adjacent nodes taking into account competition for multiple access to radio channel.
Thus, we can formulate the following mathematical statement of the problem - to find a network topology (UAV repeaters location), that minimizes the cost of transporting the data flows from sensors to gateway, when the constraints on network resources, structural connectivity and network operation parameters are performed:

$$
\begin{gathered}
X_{0}=\underset{X_{0} \in \Omega_{1,2}}{\arg \min } C(X)=\arg \min _{X_{0} \in \Omega_{1,2}} \sum_{i=1}^{N+K} \sum_{j=1}^{N+K} W_{i j}, i \neq j \\
\Omega_{1}:\left\{X \in r, p_{i j} \leq P E R^{0}, P \leq P^{0}, N \leq 1000, K \leq 100\right\} \\
\Omega_{2}:\left\{s\left(m_{a b}\right) \geq s^{0}, t_{3}\left(m_{a b}\right) \leq t_{3}^{0}, a, b=\overline{1, N}\right\},
\end{gathered}
$$

where $X$ is vector of projections coordinates of UAV set placement; $P E R^{0}$ is threshold of packet error rate in the channel; $P, P^{0}$ are network reliability and its threshold; $s\left(m_{a b}\right), t_{3}\left(m_{a b}\right), s^{0}, t_{3}^{0}$ are throughput and delay in the route $m_{a b}$ between sensor $a$ and $b$ and the corresponding thresholds.

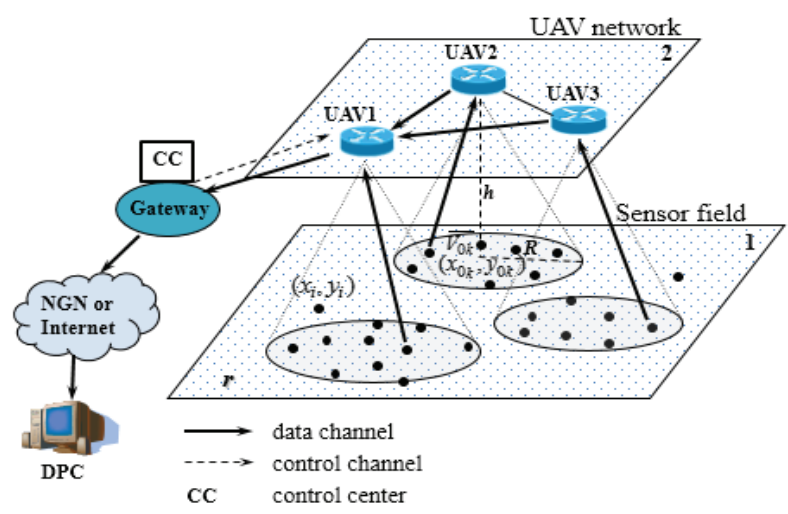

Fig.7 WSN architecture using UAVs

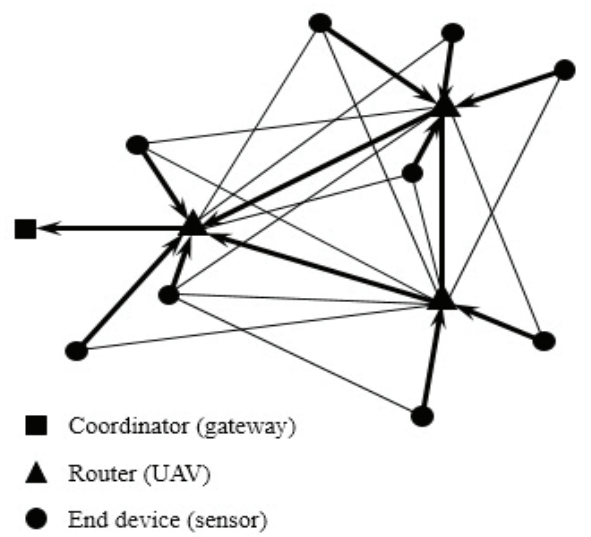

Fig.8 WSN network graph

The problem of designing a rational WSN topology from a formal point of view is similar to the problem of forming the topological structure of any wireless network, which is one of principal in its design and lies in the choice the optimal scheme of 
connection between switching units, choice of channels bandwidth and optimal routes of data transmission.

There are currently approaches to solving such problems based on the use of algorithms for combinatorial analysis. These algorithms are based on the representation of the data network in a finite graph without loops and multiple edges, whose vertices correspond to network nodes and edges links. Application of the theory of graph enumeration for solving the problem of topological optimization recently finds increasingly widespread use, which is connected with an increase in performance of computers, the development of new highly efficient algorithms for generating graphs with desired properties. The main advantage of this approach is the possibility of obtaining the exact solution for small networks therefore this approach is effective for small networks. In addition, the presence of the exact solution allows us to estimate the quality of existing and developed approaches of topology optimization.

The main disadvantage of algorithms for constructive enumeration of graphs is the inability of their application for building large-scale network topology, because the number of generated graphs grows exponentially with the growing of network nodes number. It determines the feasibility of developing an alternative approach to the design of a rational WSN topology.

To solve this problem it is developed a method, described in detail in [16], which represents a computational procedure that includes the following steps:

1) Evaluation of network connectivity. In the case of mobile sensors initially it is forecasted the duration of their stay in the radio visibility zone of UAV repeaters, and in the case of fixed - immediately it is assessed the degree of sensors coverage by UAVs repeaters. If conditions on the connectivity and network reliability is not performed, it performs the procedure of placing the UAV so that to cover all of nodes.

2) Evaluation of given functional parameters (throughput and delay of routes).

3) Improved search algorithm for a rational UAVs topology.

The essence of improvement lies in the fact that for reducing the exhaustive search of topologies, it is used a set of rules for UAVs placement that enables to find a rational solution and minimizes the time of its search. Set of rules for UAVs placement is described in detail in [16].

Thus, the application of the method will reduce the enumeration of possible graphs and get a rational solution (which differs from the optimum on $5-7 \%$ ) in real time (units / tens of seconds).

\section{Section 2 conclusion}

There was formulated a mathematical statement of the problem of finding a rational topology WSN using UAVs, which consists of a problem of sensor localization in monitoring zone and a problem of UAV relays localization in the space for the organization of connected network with desired characteristics. Rational WSN topology, built according to these principles, will reduce traffic and reduce energy consumption at the nodes, which in turn will increase network lifetime and reduce the total cost of its maintenance.

\section{References}

[1] Conti M. Mobile ad hoc networking: milestones, challenges, and new research directions / Conti M., Giordano S. // Communications Magazine, IEEE. - Vol. 52, Issue 1. - P. $85-96$.

[2] Concept of hierarchical design of intelligent control systems of MANET class tactical radio networks / [Sova O., Romanyuk V., Zhuk P., Romanyuk A.] // 22nd international conf. "UHF and telecommunication technologies" Crimico-2012. - Sevastopol: Crimico 2012. - pp. $265-266$.

[3] Romayuk V. Target functions of operational control of tactical radionetworks / Romanyuk V. // Collection of scientific work of MITI NTUU "KPI”. - 2012. - № 1. pp. $109-117$.

[4] Russel S. Artificial intelligence. A modern approach / Russel S., Norvig P. - Wiliams, 2007. - 1408 p.

[5] Bugaichenko D. Design and implementation of methods considering formal and logical specifications of self-tuning multiagent systems with time constraints: $\mathrm{PhD}$ thesis : 05.13.11 / Bugaichenko Dmitrii Yurievich. - SPb., 2007. $-259 \mathrm{p}$.

[6] Analysis of possibilities of intelligent agents usage for building the node control systems for MANET / [Sova O., Simonenko O., Romanyuk V., Umanec Y.] // Collection of scientific work of MITI NTUU "KPI". 2013. - № 1. - p. 76 - 84 .

[7] Gavrilova T. Knowledge bases of intelligent systems / Gavrilova T, Horoshevski V. - SPb.: Piter, 2000. - 384p. [8] Romanyuk V. Architecture of MANET control systems / Romayuk V., Sova O., Zhuk O. // Conf. "Problems of telecommunications - 2011". - K.:ITS NTUU “KPI” 2011. - p. 77.

[9] Minochkin A. Objective, multiagent model of operational control of mobile component of a new generation military network / Minochkin A., Shacilo P. // Collection of scientific work of MITI NTUU "KPI". 2008. - № 3. - pp. $107-118$.

[10] Minochkin A. Methods of decision making in a mobile radio network control system / Minochkin A., Romanyuk V. // Collection of scientific work of MITI NTUU “KPI". - 2006. - № 1. - pp. $66-71$.

[11] Methods of processing the knowledge about a situation in MANET network for building intelligent node control systems / [Sova O., Romanyuk V., Minochkin D., 
Romanyuk A.] // Collection of scientific work of MTI STU. - 2014. - № 1. - pp. $97-110$.

[12] Blumin S. Methods of decision making in an uncertain environment / Blumin S., Shuikova I. - Lipetsk: LEGI, 2001. - $138 \mathrm{p}$.

[13] K. Lorincz, D. J. Malan, T. R. F. Fulford-Jones, A. Nawoj, A. Clavel, V. Shnayder, G. Mainland, M. Welsh and S. Moulton, "Sensor Networks for Emergency Response: Challenges and Opportunities," Pervasive computing, 2004, Vol.10-11, pp. 16-23.

[14] M. V. Ramesh, S. Kumar and P. V. Rangan, "Wireless Sensor Network for Landslide Detection,"
International Conference on Wireless Networks (ICWN'09), Las Vegas, June 14, 2009, pp. 1-7.

[15] Z. Sun, P. Wang, M. C. Vuran, M. A. AlRodhaan, A. M. Al-Dhelaan and I. F. Akyildiz, "BorderSense: Border patrol through advanced wireless sensor networks," Ad Hoc Networks, 2011, Vol.9, pp. 468-477.

[16] O. I. Lysenko, S. V. Valuiskyi, "Capacity increasing of sensor telecommunication networks," Telecommunication Sciences, 2012, Vol.3, №1, p. 5-11.

\section{Лисенко О.І., Спаравало М.К., Романюк В.А., Валуйський С.В. Інтелектуальна система управління для WSN та MANET}

Проблематика. Запропонована нова ієрархічна модель інтелектуальної системи управління (ICS) для безпроводових сенсорних (WSN) та мобільних епізодичних мереж (MANET). Була сформульована математична постановка проблеми пошуку раціональної топології WSN 3 використанням безпілотних літальних апаратів (БПЛА), яка складається 3 проблеми локалізації сенсорів в зоні моніторингу та проблеми локалізації БПЛА ретрансляторів в просторі для організації з'язаної мережі з потрібними характеристики.

Мета досліджень. Метою статті є розробка ієрархічної моделі взаємодії інтелектуальних агентів для розвитку системи управління радіомережею MANET або WSN.

Методика реалізації. Використовувались математичні методи та алгоритми (теорія графів, методи векторної оптимізації, нечіткі алгоритми, алгоритми динамічного програмування) та комп'ютерне моделювання для описання ієрархічної моделі інтелектуальної системи управління для WSN та MANET.

Результати досліджень. Новизна моделі полягає у використанні теорії графів для формування формального опису функціональних підсистем мережі ICS (вершини графа) та процесів їх взаємодії (ребра графа). Використання запропонованої моделі дозволяє прискорити та систематизувати процес проектування мережі 3 урахуванням їх функціонуючого середовища та ієрархічної структури їх ICS.

Висновки. Використання технології інтелектуальних агентів та мультиагентних систем дозволяє мінімізувати трафік сервісу та більш ефективно використовувати мережеві та вузлові ресурси.

Ключові слова: WSN; MANET; БПЛА; управління; агент.

\section{Лысенко А.И., Спаравало М.К., Романюк В.А., Валуйский С.В. \\ Интелектуальная система управления для WSN и MANET}

Проблематика. Предложена новая иерархическая модель интеллектуальной системы управления (ICS) для беспроводных сенсорных (WSN) и мобильных эпизодических сетей (MANET). Была сформулирована математическая постановка проблемы поиска рациональной топологии WSN с использованием беспилотных летательных аппаратов (БПЛА), которая состоит из проблемы локализации сенсоров в зоне мониторинга и проблемы локализации БПЛА ретрансляторов в пространстве для организации связанной сети с нужными характеристиками.

Цель исследований. Целью статьи является разработка иерархической модели взаимодействия интеллектуальных агентов для развития системы управления радиосетью MANET или WSN.

Методика реализации. Использовались математические методы и алгоритмы (теория графов, методы векторной оптимизации, нечеткие алгоритмы, алгоритмы динамического программирования) и компьютерное моделирование для описания иерархической модели интеллектуальной системы управления для WSN и MANET.

Результаты исследований. Новизна модели заключается в использовании теории графов для формирования формального описания функциональных подсистем сети ICS (вершины графа) и процессов их взаимодействия (ребра графа). Использование предложенной модели позволяет ускорить и систематизировать процесс проектирования сети с учетом их функционирующего среды и иерархической структуры их ICS.

Выводы. Использование технологии интеллектуальных агентов и мультиагентных систем позволяет минимизировать трафик сервиса и более эффективно использовать сетевые и узловые ресурсы.

Ключевые слова: WSN; MANET; БПЛА; управление; агент. 